\title{
Heterogeneity of the action potential duration is required for sustained atrial fibrillation
}

\author{
Uma Mahesh R. Avula, ${ }^{1}$ Jeffrey Abrams, ${ }^{1}$ Alexander Katchman, ${ }^{1}$ Sergey Zakharov, ${ }^{1}$ Sergey Mironov, ${ }^{2}$ \\ Joseph Bayne, ${ }^{1}$ Daniel Roybal, ${ }^{1}$ Anirudh Gorti, ${ }^{1}$ Lin Yang, ${ }^{1}$ Vivek Iyer, ${ }^{3}$ Marc Waase, ${ }^{1}$ Deepak Saluja, ${ }^{1}$ \\ Edward J. Ciaccio, ${ }^{1}$ Hasan Garan, ${ }^{1}$ Andrew R. Marks, ${ }^{4,5}$ Steven O. Marx, ${ }^{1}$ and Elaine Y. Wan ${ }^{1}$ \\ 'Division of Cardiology, Department of Medicine, Vagelos College of Physicians and Surgeons, Columbia University, New \\ York, New York, USA. 'Center for Arrhythmia Research, Division of Cardiology, University of Michigan, Ann Arbor, Michigan, \\ USA. ${ }^{3}$ Marin General Hospital, Greenbrae, California, USA. ${ }^{4}$ The Wu Center for Molecular Cardiology, Columbia University, \\ New York, New York, USA. ${ }^{5}$ Department of Physiology and Cellular Biophysics, Vagelos College of Physicians and Surgeons, \\ Columbia University, New York, New York, USA.
}

\begin{abstract}
Atrial fibrillation (AF) is the most common cardiac arrhythmia and accounts for substantial morbidity and mortality. Recently, we created a mouse model with spontaneous and sustained AF caused by a mutation in the $\mathrm{Na}_{\mathrm{v}} 1.5$ channel (F1759A) that enhances persistent $\mathrm{Na}^{+}$current, thereby enabling the investigation of molecular mechanisms that cause AF and the identification of potentially novel treatment strategies. The mice have regional heterogeneity of action potential duration of the atria similar to observations in patients with AF. In these mice, we found that the initiation and persistence of the rotational reentrant AF arrhythmias, known as spiral waves or rotors, were dependent upon action potential duration heterogeneity. The centers of the rotors were localized to regions of greatest heterogeneity of the action potential duration. Pharmacologically attenuating the action potential duration heterogeneity reduced both spontaneous and pacinginduced AF. Computer-based simulations also demonstrated that the action potential duration heterogeneity is required to generate rotors that manifest as AF. Taken together, these findings suggest that action potential duration heterogeneity in mice and humans is one mechanism by which $A F$ is initiated and that reducing action potential duration heterogeneity can lessen the burden of AF.
\end{abstract}

Conflict of interest: EYW previously received financial compensation for speaking at Abbott and Medtronic teaching symposia. ARM is the founding scientist and chair of the Scientific Advisory Board of ARMGO Pharma, Inc., which is targeting leaky ryanodine receptor channels for treatment of cardiovascular and skeletal muscle disorders, including atrial fibrillation. Both ARM and Columbia University own equity in ARMGO.

Copyright: (c) 2019 American Society for Clinical Investigation

Submitted: March 11, 2019

Accepted: April 17, 2019

Published: June 6, 2019.

Reference information: JCl Insight. 2019;4(11):e128765. https://doi. org/10.1172/jci.insight.128765.

\section{Introduction}

Atrial fibrillation (AF) is the most common cardiac arrhythmia, yet its mechanisms are still unclear and therapeutic options are limited. The development of AF is a complex process involving interplay between electrical and structural remodeling in the atria, autonomic imbalance, abnormal metabolism, and genetic factors. In humans, genetic and acquired $\mathrm{Na}^{+}$channel dysfunctions have been associated with cardiomyopathy and electrical instability, including AF (1-3). Aging, atrial stretch, and fibrosis also alter the anatomical substrate by increasing persistent $\mathrm{Na}^{+}$current (4), thereby prolonging the action potential duration (APD) and subsequently triggering arrhythmogenesis. Drugs that reduce persistent $\mathrm{Na}^{+}$current are frequently used to treat patients with AF. Recently, we reported that increased persistent $\mathrm{Na}^{+}$current, caused by transgenic (TG) expression in mice of a gain-of-function, mutant human $\mathrm{Na}_{\mathrm{v}} 1.5$ channel, was sufficient to cause an atrial cardiomyopathy and prolonged episodes of spontaneous AF (5).

In humans, the initiation of $\mathrm{AF}$ is frequently triggered by increased automaticity within the myocardial sleeves of pulmonary veins. However, isolating the pulmonary veins by ablation is often not curative because AF can also be initiated by triggers outside the pulmonary veins, for example, within the posterior atrial wall (6). The discovery of electrical rotational activity in the atria, called rotors or spiral waves, shown using high-resolution optical mapping in animal models of AF, and activation mapping in humans with multi-electrode, intra-atrial basket catheters (7) and body surface electrograms has driven new ablative therapeutic approaches. However, the question remains: what determines the location of a rotor? 
Atrial fibrosis has been proposed as one mechanism for rotor clustering, and whereas a correlation between the amount of late gadolinium enhancement detected by MRI and the number of regions exhibiting reentry has been shown (8), other studies have found no such correlation $(9,10)$. A role for APD heterogeneity in the genesis of rotors has been shown in in vitro cell culture studies of virally transduced ventricular myocyte monolayers variably expressing the hERG (human ether-a-gogo-related gene) protein in neonatal rats. The predominant areas of rotational activity and wavebreaks were in border zones of enhanced APD dispersion (11). Computer simulations have shown that areas of greatest APD dispersion and refractoriness, leads to wavebreak and initiation of reentry, explaining the progression from sinus rhythm or rapid tachycardia to AF $(12,13)$. These conclusions have yet to be tested in vivo because a limitation of both large- and small-animal models of AF is the inability to recapitulate the spontaneity and long duration of AF seen in humans. Previous studies in rabbits, have shown APD differences, but not intra-atrial APD dispersion (14).

Herein, we show that in a mouse model of spontaneous AF, rotors and reentrant waves are dependent on atrial APD heterogeneity and are anchored to regions where the APD dispersion is greatest. Using a monophasic action potential (MAP) contact catheter (15), we show that spatial APD heterogeneity is similarly present in the posterior left atrial wall of patients with paroxysmal or persistent AF. Attenuating the atrial APD heterogeneity reduces spontaneous and pacing-induced AF re-induction in mice. Taken together, these in vivo and in silico studies (16) suggest that spatial APD dispersion is one mechanism by which $\mathrm{AF}$ is initiated and can anchor rotational reentry.

\section{Results}

Wavebreaks and reentrant waves in AF mice. The AF mice were generated by crossing mice with FLAG-tagged F1759A human $S C N 5 A\left(\mathrm{Na}_{\mathrm{v}} 1.5\right)$ fused to a modified murine $\alpha$-myosin heavy chain, tetracycline-inducible promoter vector (17) and mice with cardiac-specific expression of reverse tetracycline-controlled transactivator protein (rtTA) (18). Without doxycycline, the double-transgenic (dTG) mice developed structural alterations, including atrial and ventricular enlargement, myofibril disarray, fibrosis, mitochondrial necrosis, and electrophysiological dysfunctions, leading to spontaneous and prolonged episodes of AF (5) (Figure $1 \mathrm{~A}$ ). The expression of the F1759A-Na 1.5 channels in the absence of doxycycline is likely due to a low basal binding of rtTA protein to the Tet operator sequences (called "leak") (18). Not only did the F1759A mutation (19) enable us to distinguish the functional characteristics of the $\mathrm{TG} \mathrm{Na}^{+}$channels, which are relatively resistant to local anesthetics such as lidocaine, from endogenous channels, but it also prevented complete inactivation of $\mathrm{Na}_{\mathrm{v}} 1.5$, resulting in a persistent $\mathrm{Na}^{+}$current.

Epicardial surface optical voltage mapping of the anterior surface of Langendorff-perfused F1759A-dTG hearts revealed a variety of activation patterns, including a single dominant clockwise or counterclockwise rotor, figure of 8 reentry, multiple rotors in either atrium or both atria, and multiple wavebreaks with fibrillatory conduction (Figure 1B and Supplemental Figure 1A; supplemental material available online with this article; https://doi.org/10.1172/jci.insight.128765DS1). Rotors are spiral waves with patterns of circular reentry for 1 or more cycles. AF persisted with the same arrhythmic mechanisms (rotors or wavebreaks) during extended recordings of up to 1 hour. Conduction velocity and the dispersion of the conduction velocity were not significantly altered in the TG mice compared to littermate controls (Supplemental Figure 1, B-G)

To elucidate the underlying electrophysiological substrate, the Langendorff-perfused hearts were perfused with a hyperkalemic solution to terminate the arrhythmias. Thereafter, a normokalemic solution was infused, and the atrial APD was measured by pacing the atria at $10 \mathrm{~Hz}$ (Figure 1, C and D). The maximal and mean APD were increased by approximately 2-fold in both right and left atria of the F1759A-dTG mice compared with control mice (Figure 1E). Consistently, we observed APD heterogeneity in both left and right atria - demonstrated by the nonuniformity in APD maps (Figure 1C) and in all-point histograms of the APD (Figure 1F). The dispersion of APD, assessed by both the difference between greatest and least APD (Figure 1G) and the coefficient of variation of APD (Supplemental Figure 1, F and G), was significantly greater in the F1759A-dTG mice compared with littermate control mice.

Heterogeneity of APD in patients with $A F$. Does spatial heterogeneity of the APD exist in patients with $\mathrm{AF}$ ? Prior recordings of MAP studies in humans demonstrated that alternans of the APD is a dynamic substrate for AF (20). We acquired electro-anatomical voltage maps and MAPs in sinus rhythm in 5 
A
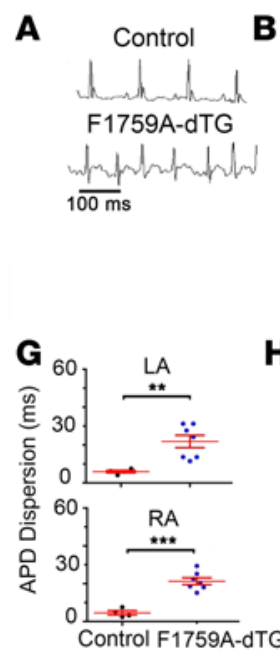

H
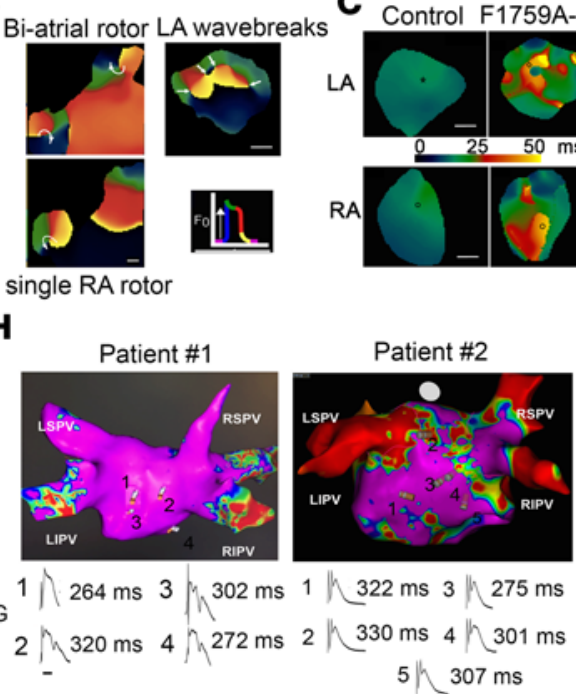

single RA rotor
C Control F1759A-dTG D
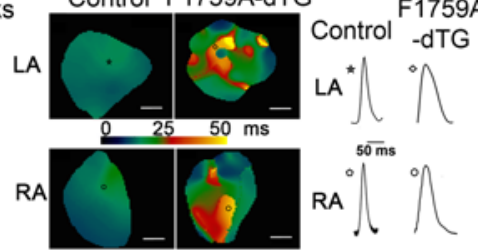

F1759A

-dTG
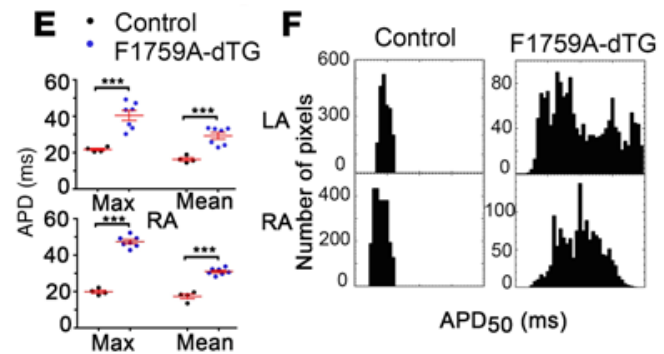

$\mathrm{APD}_{50}$ (ms)

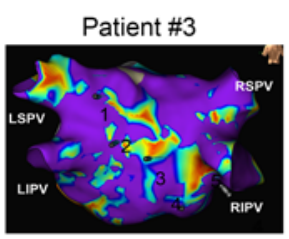

1 h $322 \mathrm{~ms} 3275 \mathrm{~ms}$

2 330 ms 4 \301 ms

$5 \bigwedge 307 \mathrm{~ms}$

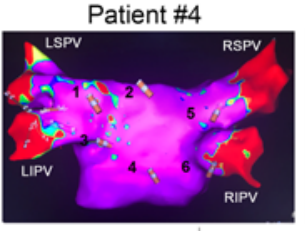

$1 \mathrm{~N}^{314 \mathrm{~ms}} 4 \mathrm{~h}^{315 \mathrm{~ms}} 1 \mathrm{~h}^{268 \mathrm{~ms}} 3 h^{338 \mathrm{~ms}}$

$2 \mathrm{~h} 340 \mathrm{~ms} 5 \mathrm{~h} 371 \mathrm{~ms} 2 \mathrm{~m} 275 \mathrm{~ms} 4 \mathrm{hn}^{280 \mathrm{~ms}}$

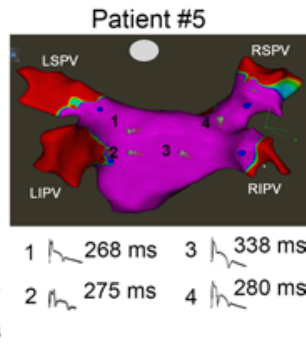

3 M $364 \mathrm{~ms} 6 \mathrm{~m}, 323 \mathrm{~ms}$

Figure 1. Inhomogeneity of APD in mice and humans with AF. (A) Representative limb lead surface ECGs of isoflurane-anesthetized littermate control mouse in sinus rhythm (top row) and of F1759A-dTC mouse in AF (lower row). (B) Representative snapshots from phase movies of Langendorff-perfused F1759A-dTC hearts demonstrating simultaneous rotors in the right atrium (RA) and left atrium (LA), a predominant rotor in the RA, and wavebreaks and fibrillatory conduction in the LA. (C and D) Representative optical APD maps (C) and optical action potential tracings (D) from littermate control and F1759A-dTC mice. APD maps (pacing at $10 \mathrm{~Hz}$ ) for F1759A-dTC mice were obtained after hyperkalemia-induced conversion to sinus rhythm. The circle marks the region corresponding to the optical action potential tracings in D. Scale bar: $1 \mathrm{~mm}$. (E) Graph showing maximal (max) and mean APD ${ }_{50}$ in $L_{A}$ and RA of littermate control $(n=4)$ and F1759A-dTG mice $(n=7)$. Mean \pm SEM. ${ }^{* *} P<0.001$; 2-tailed Student's $t$ test. (F) Representative all-points histograms of APD. (C) Graphs of $\mathrm{APD}_{50}$ dispersion. Mean $\pm \mathrm{SEM}$ for littermate control and F1759A-dTC mice. ${ }^{* *} P<0.01$; ${ }^{* * *} P<0.01 ; 2$-tailed Student's $t$ test. (H) Electro-anatomical voltage map (upper) and MAP recordings in sinus rhythm (lower) with $\mathrm{APD}_{90}$ measurement for the corresponding regions for 5 patients undergoing AF ablation. For the electro-anatomical voltage map, red color $(0.2 \mathrm{mV})$ is indicative of low-voltage area consistent with scarred tissue, and purple (0.5-1.0 mV) is indicative of normal healthy tissue. LIPV, linferior pulmonary vein; RIPV, right inferior pulmonary vein; LSPV, eft superior pulmonary vein; and RSPV: right superior pulmonary vein.

patients with paroxysmal or persistent $\mathrm{AF}$ and normal left ventricular function. The patients ranged in age from 35 to 85 with a mean of $62.4 \pm 8.4$ years, $80 \%$ were men, BMI was $28.5 \pm 3.4 \mathrm{~kg} / \mathrm{m}^{2}$, and LA diameter was $3.9 \pm 0.2 \mathrm{~cm}$. In patients $1,3,4$, and 5 , electro-anatomical voltage mapping revealed little to mild scarring of the posterior wall, whereas patients 2,4 , and 5 showed scarring within the pulmonary veins (Figure $1 \mathrm{H})$. We selected 4 to 6 regions without scarring in each patient to measure the APD at $90 \%$ repolarization during atrial pacing at a cycle length of $500 \mathrm{~ms}$ (120 beats per minute). The dispersion of APD in the 5 patients, assessed by the difference between the greatest and least $\mathrm{APD}$, was $39,55,56,57$, and $70 \mathrm{~ms}$, respectively. We were unable to perform similar mapping studies on control patients without a history of atrial arrhythmias because of IRB restrictions. Although we cannot exclude the possibility that similar heterogeneity is present in patients without AF, the most conservative interpretation of the human findings is that heterogeneity of the APD exists in patients with AF, perhaps enabling the sustenance of AF. Using our mouse model of AF, we sought to identify the role of heterogeneity of AF in the initiation and perpetuation of AF.

Spatial APD gradients are a substrate for reentry, rotors, and wavebreaks. Using both anti-FLAG immunocytochemistry (Figure 2, A and B) and patch clamp (Figure 2, C-F) analyses of isolated atrial cardiomyocytes, we found that expression of the FLAG epitope-tagged F1759A-mutant $\mathrm{Na}_{\mathrm{v}} 1.5$ channels varied from cell to cell in the F1759A-dTG mice. Some myocytes had no lidocaine-resistant $\mathrm{Na}^{+}$current, whereas others had more than $60 \%$ resistant current (Figure 2, C and D). The F1759A mutation prevented complete inactivation of $\mathrm{Na}_{\mathrm{v}} 1.5$, thereby increasing persistent $\mathrm{Na}^{+}$current in atrial cardiomyocytes isolated from F1759A-dTG mice (Figure 2E). Although the F1759A-Na 1.5 channels are relatively resistant to ranolazine, the persistent $\mathrm{Na}^{+}$current is readily inhibited by superfusion of 500 $\mu \mathrm{M}$ ranolazine (Figure $2 \mathrm{E}$ ). Similar to and directly correlated with the variation in lidocaine-resistant $\mathrm{Na}^{+}$current density, we observed marked variation in the amount of persistent $\mathrm{Na}^{+}$current caused by variable cell-to-cell expression of F1759A-Na 1.5 channels in atrial cardiomyocytes (Figure $2 \mathrm{~F}$ ). The cell-to-cell variation in expression of the F1759A-Na 1.5 channels in the absence of doxycycline is like- 
A
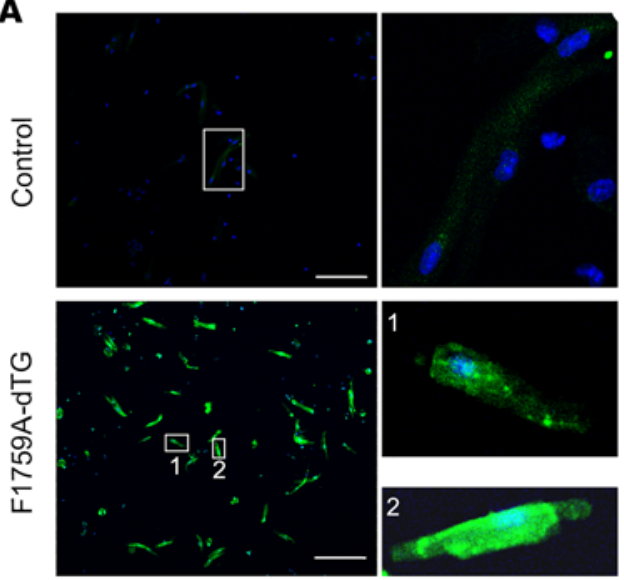

C
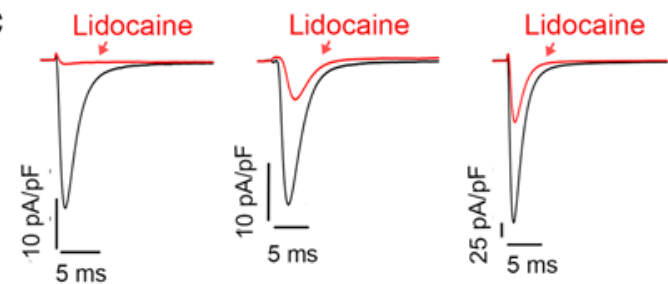

$\mathbf{E}$
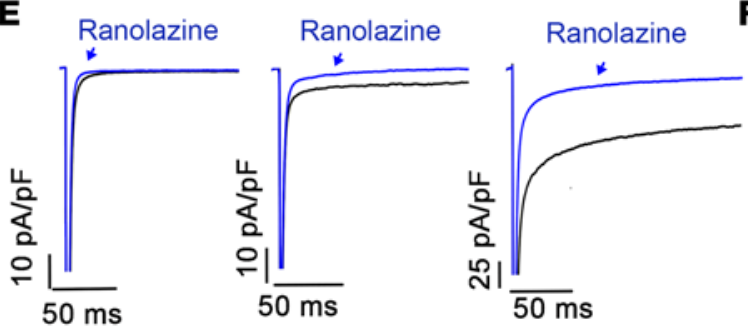

B

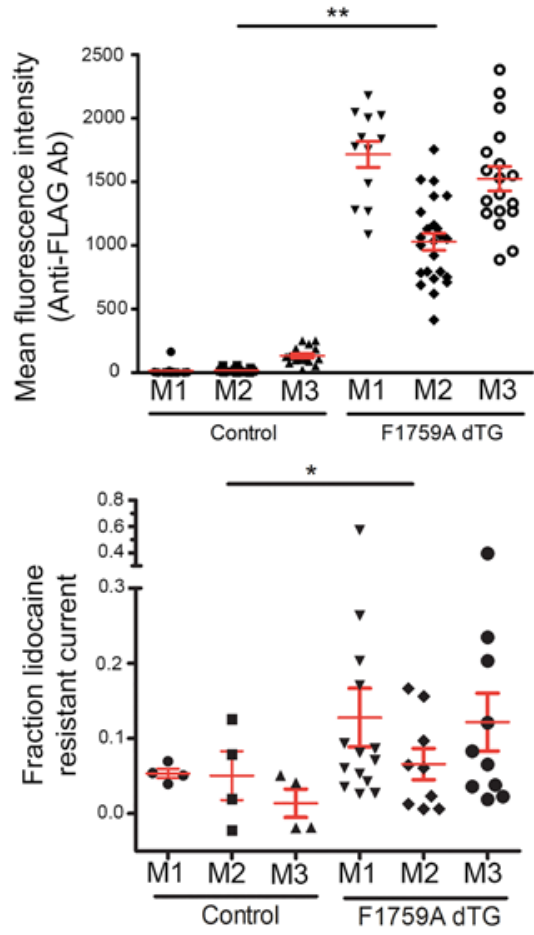

$\mathbf{F}$

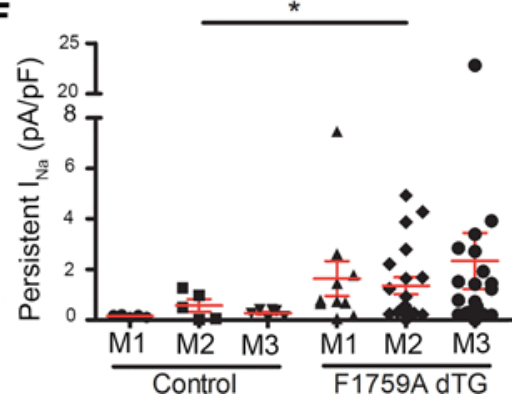

Figure 2. Inhomogeneity of TG $\mathrm{Na}_{\mathrm{v}} \mathbf{1 . 5}$ expression and persistent $\mathrm{Na}^{+}$current in F1759A-dTG mice. (A) Representative immunofluorescent images of atrial cardiomyocytes isolated from littermate control mice and F1759A-dTC mice. Atrial cardiomyocytes were permeabilized and incubated with or without anti-FLAG antibody and with FITC-conjugated secondary antibody. Images were obtained with confocal microscopy at $\times 20$ (left) and $\times 40$ original magnification (right). Scale bars: $100 \mu \mathrm{m}$. (B) Graph quantifying immunofluorescent intensity using ImageJ (NIH). Mean \pm SEM. ${ }^{* *} P<0.01$, 2-tailed Student's $t$ test; $n=3$ mice for each group, control and F1759A-dTC. M1, mouse 1; M2, mouse 2; M3, mouse 3. (C) Exemplar whole-cell $\mathrm{Na}^{+}$current $\left(I_{\mathrm{Na}}\right)$ traces of atrial cardiomyocytes isolated from F1759A-dTC mice. Whole-cell current traces were recorded with $5 \mathrm{mM} \mathrm{Na}^{+}$in both extracellular and intracellular solutions, in the absence (black) and presence (red) of $3 \mathrm{mM}$ lidocaine. (D) Fraction of lidocaine-resistant current for littermate control. Mean \pm SEM; $n=3$ mice for each group, control and F1759A-dTC. M1, mouse 1; M2, mouse 2; M3, mouse 3. (E) Exemplar whole-cell $\mathrm{Na}^{+}$current traces designed to assess persistent $\mathrm{I}_{\mathrm{Na}}$ using a 190-ms depolarization from a holding potential of -110 to $-30 \mathrm{mV}$ in the absence (black) and presence (blue) of $500 \mu \mathrm{M}$ ranolazine; intracellular solution contained $5 \mathrm{mM} \mathrm{Na}^{+}$and extracellular solution contained $100 \mathrm{mM} \mathrm{Na}^{+}$. $n=3$ mice; $n=54$ cardiomyocytes. (F) Graph of extent of persistent $\mathrm{I}_{\mathrm{Na}}$. Mean \pm SEM. $n=3$ mice for each group, control and F1759A-dTG, ${ }^{* *} P<0.05$; 2-tailed Student's $t$ test. M1, mouse 1; M2, mouse 2; M3, mouse 3.

ly due to variations in the leakiness of the Tet promoter in the atrial myocytes.

Why are rotors and wavebreaks anchored in specific regions of the atria, and what, if any, electrophysiological restraints dictate the meandering boundaries? The core of the rotor, known as a singularity point, and areas of wavebreaks were quantified by plotting the singularity points over time and creating singularity point density (SPD) maps for both spontaneous AF and AF that was re-initiated either spontaneously or by burst pacing after hyperkalemia-induced conversion. The trajectories of the singularity points then were superimposed on 2-dimensional and 3-dimensional projections of the $\mathrm{APD}_{50}$ map acquired in sinus rhythm. For both spontaneous and re-initiated AF, the trajectory of the 
rotor's core meandered but was tightly delimited within the regions of high APD gradients (Figure 3, A and B, and Supplemental Figure 2, A and B). Similar patterns were observed in hearts with a dominant rotor (Supplemental Video 1) and hearts with multiple wavebreaks (Supplemental Video 2) in the atria. In 10 of 11 mice with spontaneous AF, the APD dispersion and the density of singularity points were positively and significantly correlated (Figure $3 \mathrm{C}$ ), with a mean slope of $4.0 \pm 0.7$ singularity points/ms of APD dispersion $(P<0.0001)$.

In regions with a high density of singularity points, the mean APDs were increased by approximately 2-fold (Figure 3D), and the APD dispersion was increased by approximately 3- to 4-fold in the atria of F1759A-dTG mice as compared with similar locations in littermate control mice (Figure 3E). The APD dispersion within regions of high density of singularity points, determined by subtracting the maximal and minimal APD values within the small $10 \times 10$ pixel box, was nearly as high as the dispersion throughout each atrium. We quantified the dependence of rotor and wavebreak anchoring upon heterogeneity of APD by manually determining within multiple $10 \times 10$ pixel boxes the maximal APD dispersion and the density of singularity points during each of the 4096 frames of a 5 -second optically acquired movie. Overall, these findings imply that the singularity points of rotors and the regions of wavebreaks are anchored at regions of high APD gradients, specifically at the boundaries of long and short APD regions.

Minimizing spatial APD gradients reduces $A F$ inducibility. Consistent with the thesis that increased APD causes early afterdepolarizations (EADs) that can trigger arrhythmias, we observed using optically acquired voltage maps and time-space plots pacing-induced phase 3 EADs and rotors in the atria of the F1759A-dTG hearts (Figure 4, A-D). Previously, we showed that a single intraperitoneal injection of SEA-0400, an inhibitor of the $\mathrm{Na}^{+} / \mathrm{Ca}^{2+}$ exchanger, markedly reduced the burden of spontaneous AF in mice (5). We optically acquired voltage maps of Langendorff-perfused F1759A-dTG hearts before (vehicle) and after perfusion with SEA-0400. SEA-0400 did not significantly alter atrial $\mathrm{APD}$ or APD dispersion (Figure 4, E and F); however, the number of EADs and episodes of AF induced by EADs were markedly reduced (Figure 4, G-I). Thus, the initiation or persistence of AF in this model requires the generation of EADs.

We also hypothesized that inhomogeneity of the atrial APD can form the substrate for initiating and sustaining atrial arrhythmias in these mice. We optically acquired voltage maps of Langendorff-perfused hearts before and after infusion of $500 \mu \mathrm{M}$ ranolazine and $20 \mathrm{nM}$ anemone toxin (ATX-II) (Figure 5, A-C). Ranolazine was sufficient to reduce the APD by $30 \%(P<0.0001)$ and APD dispersion by $50 \%$ $(P<0.0001)$ compared with vehicle-perfused hearts (Figure 5, A, B, and D). Ranolazine had potent antifibrillatory effects: whereas sustained AF was induced by $20-\mathrm{Hz}$ pacing in $100 \%$ of vehicle-perfused hearts, none of the ranolazine-perfused hearts could be induced to AF ( $n=5$ animals; $P=0.008$ by Fisher's exact test). Although ranolazine markedly reduced the number of EADs (Figure 5, E and F), likely predominantly accounting for the antiarrhythmic effect, the likelihood of EADs causing AF was also completely reduced by ranolazine (Figure 5F). Ranolazine had minimal effects on conduction velocity (Supplemental Figure 3). Although an off-target effect of ranolazine is inhibition of hERG channels (21), these channels are not expressed in murine myocardium.

The converse approach for reducing APD dispersion is to increase the APD throughout the atria. ATX-II, which enhances persistent $\mathrm{Na}^{+}$current $(22)$, increased the mean atrial APD by $31 \%(P<0.0001)$ but concomitantly reduced APD dispersion by $38 \%(P<0.001)$ (Figure $5, \mathrm{C}$ and D). ATX-II also had potent antifibrillatory effects: only 1 of 5 mice had inducible AF by $20-\mathrm{Hz}$ burst pacing $(P=0.048$ by Fisher's exact test compared with control). The conduction velocity, however, was only minimally affected by ATX-II (Supplemental Figure 3). Although ATX-II did not reduce the frequency of EADs, the frequency of EADs triggering AF was markedly reduced (Figure 5, F and G).

Next, we explored whether afterdepolarizations in the substrate of normal APD and normal dispersion of APD could initiate and sustain AF in the F1759A-dTG hearts. Langendorff-perfused F1759A-dTG hearts were first treated with ranolazine, which normalized the APD and the dispersion of APD (Figure 5, H and I). To increase afterdepolarizations, we added $0.9 \mu \mathrm{M}$ digoxin, a $\mathrm{Na}^{+} /$ $\mathrm{K}^{+}$-ATPase inhibitor, to the ranolazine-containing perfusate. Although digoxin had no effect on the APD or dispersion of the APD (Figure 5, H and I), it markedly increased the number of afterdepolarizations (Figure 5, $\mathrm{J}$ and $\mathrm{K}$ ), but these afterdepolarizations failed to initiate or perpetuate $\mathrm{AF}$ in the presence of ranolazine (Figure 5L). Taken together, these findings demonstrate that spatial APD 
A
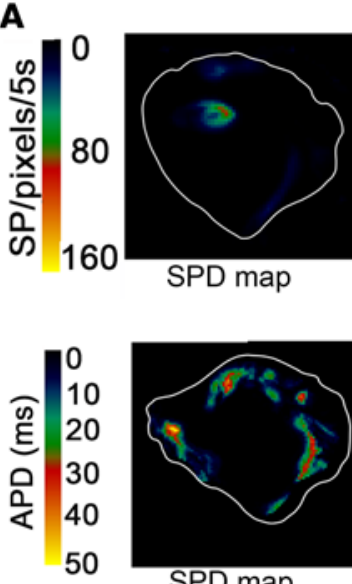

SPD map

B

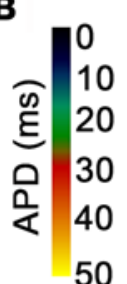

50

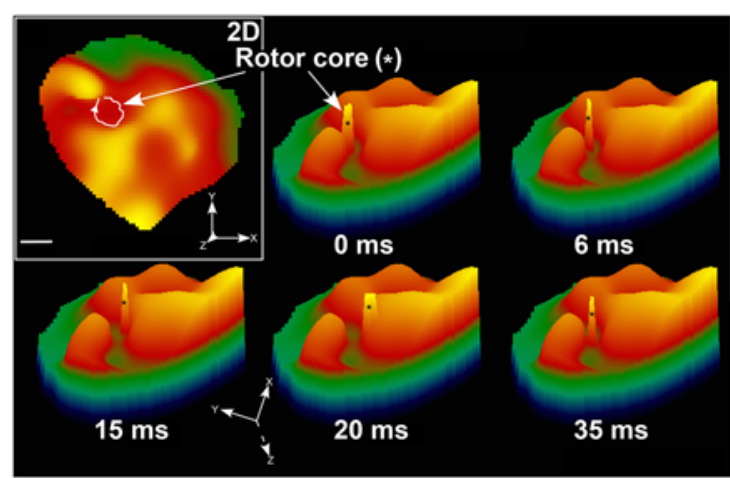

Rotors
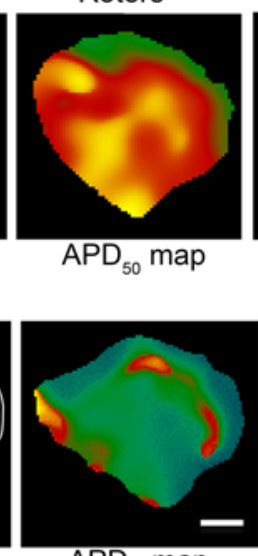

$\mathrm{APD}_{50}$ map

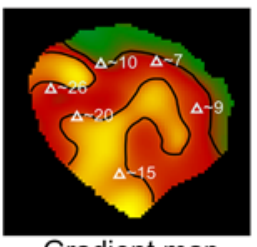

Gradient map

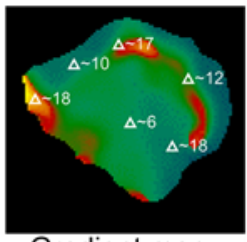

Gradient map

\section{C}

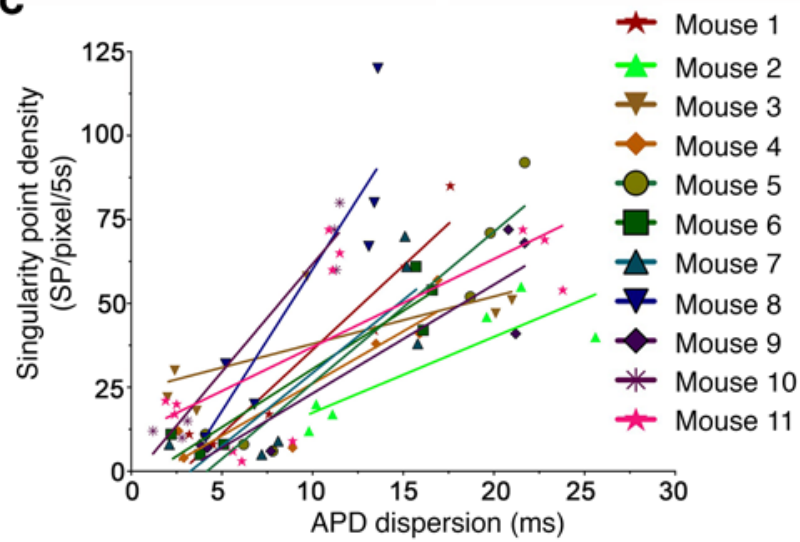

D

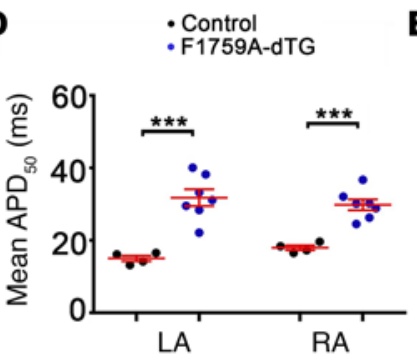

E

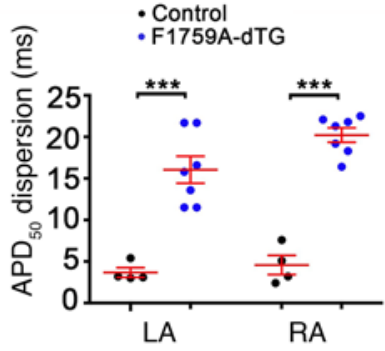

Figure 3. Rotors are anchored in regions of high spatial APD inhomogeneity. (A) Representative SPD maps and corresponding $\mathrm{APD}_{50}$ maps (middle) of single rotor (upper) and wavebreaks (lower) from F1759A-dTC mice. In the gradient APD $_{50}$ map (right), differences in APD $_{50}$ between adjacent segments are shown on the contoured gradient map ( $\Delta$ in ms). Note alignment of high density of singularity points and dispersion of APD. Scale bar: $1 \mathrm{~mm}$. (B) Representative 2-dimensional and 3-dimensional images of the APD ${ }_{50}$ within the LA of a F1759A-dTC mouse. The positions of the rotor core at different time points are overlaid on the $A P D_{50}$ maps. The trajectory of the rotor core is delimited within the regions of high spatial APD gradients. (C) Scatter plot depicting correlation between APD dispersion and SPD. Lines are best fit. Mouse 1: $R^{2}=0.92, P<0.01$; mouse 2: $R^{2}=0.75, P<0.05 ;$ mouse $3: R^{2}=0.56, P=0.09$; mouse 4: $R^{2}=0.81, P<0.05$; mouse 5: $R^{2}=0.91, P<0.01$; mouse 6: $R^{2}=0.90, P<0.01$; mouse 7: $R^{2}=0.72, P<0.05$; mouse 8: $R^{2}=0.81, P<0.05 ;$ mouse 9 : $R^{2}=0.85, P<0.01$; mouse 10: $R^{2}=0.95, P<0.001$; mouse 11: $R^{2}=0.54, P<0.01$. (D) Graph showing mean $\mathrm{APD}_{50}$ at the maximal SPD position in the left and right atria of F1759A-dTC mice. Mean \pm SEM. ${ }^{* * *} P<0.001$ by 2-tailed Student's $t$ test. (E) Graph of $A^{*} D_{50}$ dispersion at the maximal SPD position in the left and right atria of F1759A-dTC mice. Mean \pm SEM. ${ }^{* *} P<0.001$ by 2 -tailed Student's $t$ test.

inhomogeneity is essential for initiating and sustaining AF caused by increased persistent $\mathrm{Na}^{+}$current.

Simulations demonstrate that spatial APD heterogeneity promotes $A F$. We used a previously described automaton (16) to test whether spatial APD heterogeneity is sufficient to perpetuate AF. In this simulation, we used 2 APD values, 100 and $130 \mathrm{~ms}$, and induced fibrillatory activity by delivering premature $\mathrm{S}_{1}-\mathrm{S}_{2}$ stimulation. When the grid had a homogenous APD (Figure $5 \mathrm{M}$ ), extrastimuli did not produce any fibrillatory activity. In contrast, when the grid contained 2 APD regions with a soft boundary between the 2, extrastimuli caused sustained rotors with the singularity point fixed at the boundary between the 2 APD regions (Figure 5M, Supplemental Video 3). Thus, similar to previously published modeling studies $(11,23,24)$, we found that heterogeneity of the APD is sufficient to form a suitable substrate to perpetuate AF.

\section{Discussion}

There are several lines of evidence supporting the relevance of this $\mathrm{AF}$ model and gain-of-function $\mathrm{Na}_{\mathrm{v}} 1.5$ abnormalities to AF in humans (25): (a) the incidence of AF is increased in patients with long QT syndrome type 3 who harbor SCN5A mutations (26); (b) persistent $\mathrm{Na}^{+}$current is increased by $26 \%$ in atrial appendages of patients with permanent AF (2); (c) ranolazine reduced atrial arrhythmias and new AF episodes in clinical trials $(27,28)$; and (d) increased persistent $\mathrm{Na}^{+}$current is observed in pathological conditions associated with an increased incidence of AF. Naturally, mouse models have limitations, espe- 
A

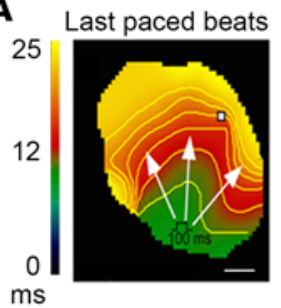

C<smiles>c1ccccc1</smiles>

EAD triggered beats

LV

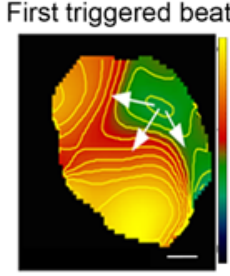

B

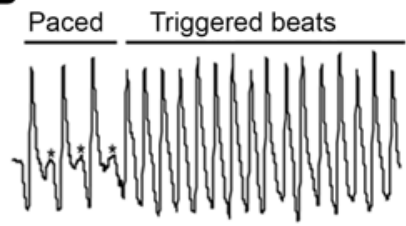

E
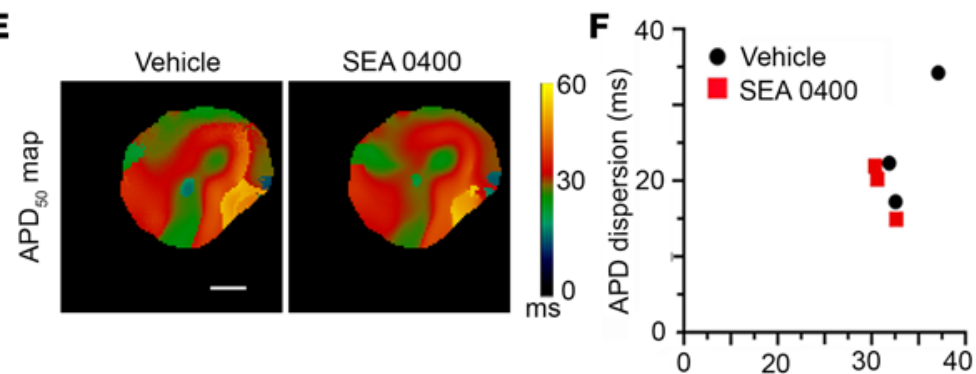

G

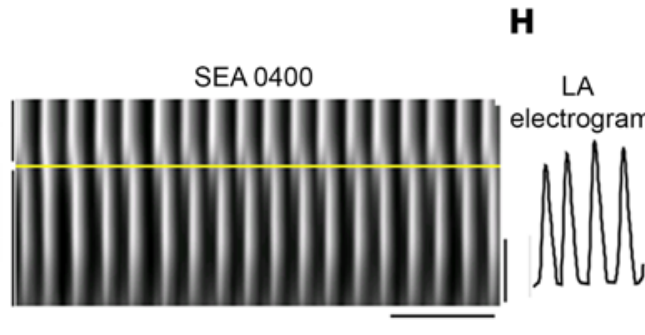

D LA electrogram

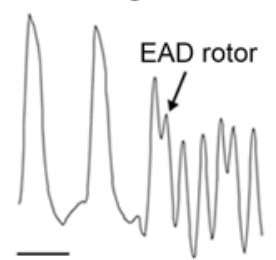

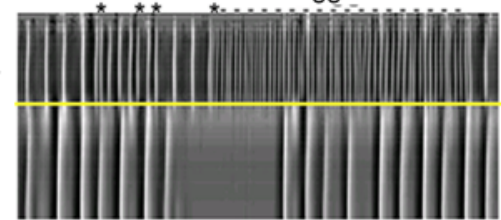

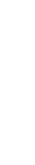


A

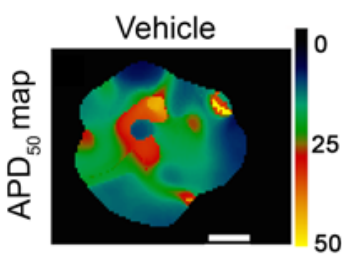

B

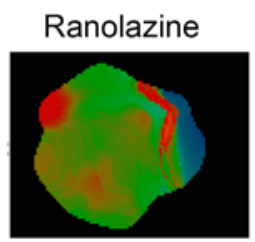

C

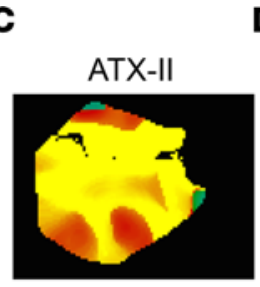

D

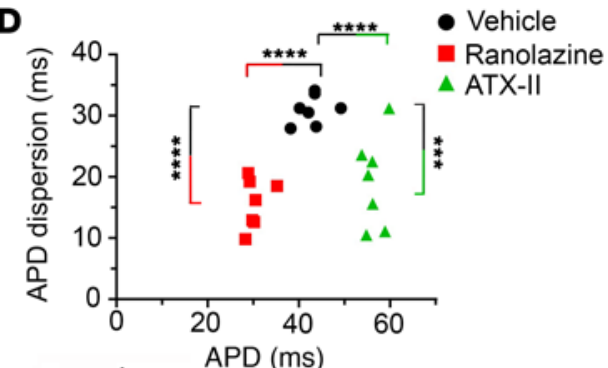

E

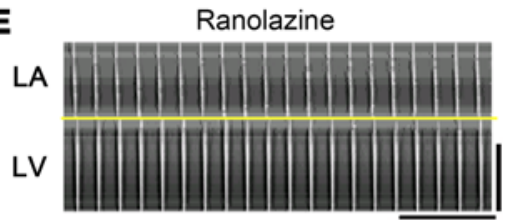

G

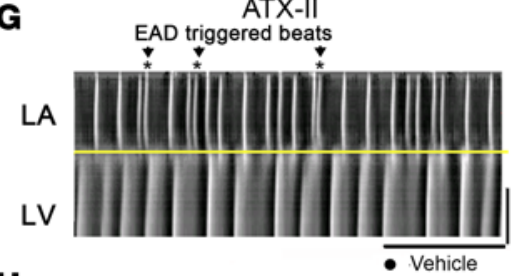

\begin{tabular}{l} 
H \\
$\frac{0}{0}$ \\
$\frac{0}{\frac{D}{0}}$ \\
\hline
\end{tabular}
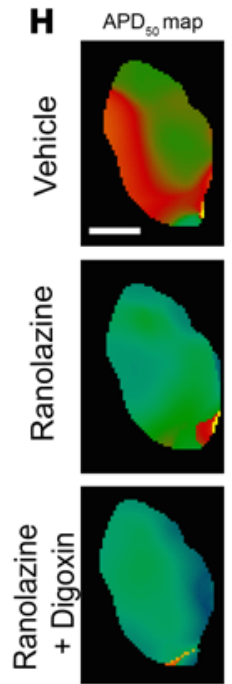$$
\text { I }
$$

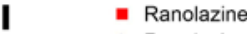

A Ranolazine + Digoxin

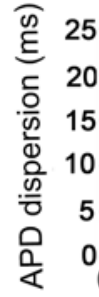

$$
\text { L }
$$$$
\text { 닌 }
$$$$
\text { 동. }
$$$$
\text { 政 }
$$
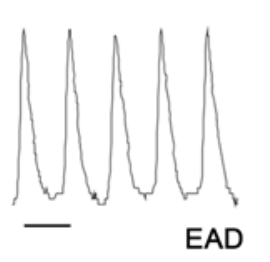

F

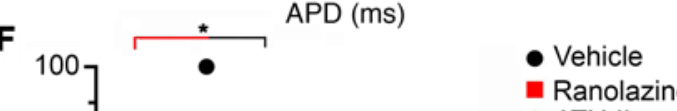

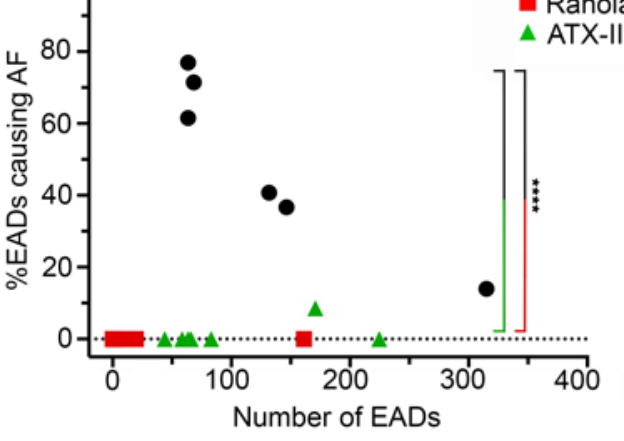

J

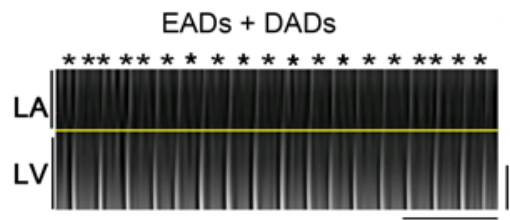

K $E A D s+D A D s$

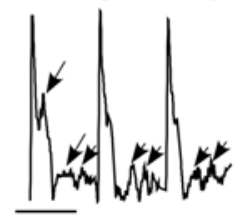

APD

M
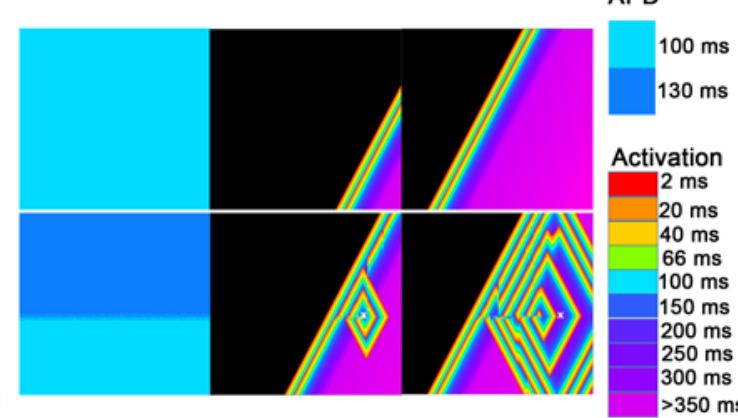

Figure 5. Inhomogeneity of the APD is required for AF in F1759A-dTG mice. (A-C) Representative $A P D_{50}$ maps in sinus rhythm before (vehicle), after 500 $\mu \mathrm{M}$ ranolazine, and after $20 \mathrm{nM}$ ATX-II. Scale bar: $1 \mathrm{~mm}$. (D) Graph depicting relationship between APD and APD dispersion before (vehicle) and after either $500 \mu \mathrm{M}$ ranolazine or $20 \mathrm{nM}$ ATX-II. ${ }^{* *} P<0.001 ;{ }^{* * *} P<0.0001$ by 1 -way ANOVA and Dunnett's multiple-comparisons test. The color and direction of the brackets indicate the pair of comparisons. (E) Representative time-space plot of LA and LV during $10-\mathrm{Hz}$ atrial pacing after $500 \mu \mathrm{M}$ ranolazine. Scale bar: $500 \mathrm{~ms}$. (F) Graph depicting relationship between number of EADs/min and percentage of EADs causing AF before (vehicle) and after either ranolazine or ATX-II. ${ }^{*} P<0.05$ and ${ }^{* * *} P<0.0001$ by 1-way ANOVA and Dunnett's multiple-comparisons test. The color and direction of the brackets indicate the pair of comparisons. (C) Representative time-space plot of LA and LV during $10-\mathrm{Hz}$ atrial pacing after $20 \mathrm{nM}$ ATX-II. EADs are marked by *. Horizontal scale bar: 500 ms. Vertical scale bar: 2.5 mm. Electrogram shows EADs. Scale bar: 100 ms. (H) Representative APD ${ }_{50}$ maps in sinus rhythm before (vehicle), after 500 $\mu \mathrm{M}$ ranolazine, and after ranolazine and $0.9 \mu \mathrm{M}$ digoxin. (I) Graph depicting relationship between APD and APD dispersion before (vehicle) and after either $500 \mu \mathrm{M}$ ranolazine or $500 \mu \mathrm{M}$ ranolazine and $0.9 \mu \mathrm{M}$ digoxin. ${ }^{* *} P<0.001 ;{ }^{* * *} P<0.0001$ by 1-way ANOVA and Dunnett's multiple-comparisons test. The color and direction of the brackets indicate the pair of comparisons. (J) Representative time-space plot of LA and LV during 10-Hz atrial pacing after ranolazine and digoxin. Afterdepolarizations are marked by asterisks. (K) Electrogram shows EADs and DADs. (L) Graph depicting relationship between number of afterdepolarizations/min and percentage of afterdepolarizations causing AF before (vehicle) and after either ranolazine or ranolazine and digoxin. The color and direction of the brackets indicate the pair of comparisons. (M) Automaton simulation of fibrillatory activity in atrial tissue. (Upper) A uniform APD gradient of 100 ms was imposed. (Lower) Two APDs were imposed: $100 \mathrm{~ms}$ and $130 \mathrm{~ms}$. Electrical activation was initiated by an $\mathrm{S}_{1}-\mathrm{S}_{2}$ pulse with 84 -ms coupling interval from the lower right-hand grid corner (node set to state 1 for each pulse; see Methods). No reentry was seen in a homogeneous uniform gradient. In the nonuniform simulation, activation by an $\mathrm{S}_{1}-\mathrm{S}_{2}$ pulse caused fibrillatory activity in the form of rotors at the boundary between the 2 APD gradients of 100 and $130 \mathrm{~ms}$. 
cellular basis for MAPs $(15,29)$. It is unlikely, however, that the inhomogeneity of $\mathrm{APD}_{90}$ measured by MAP contact electrode was artifactual, caused by catheter movement or variability in contact force, because the MAP signals were stable and of typical and consistent morphology. Is the inhomogeneity of the APD abnormal in patients with AF? Because of IRB restrictions, we could not determine whether inhomogeneity of the APD is also present in patients without AF. Some degree of dispersion may be present in the normal LA. It is conceivable that individuals without AF also have inhomogeneity of the APD, perhaps predisposing them to AF over time, especially if they develop diseases associated with increased EADs (for example, heart failure). In rabbits without disease, for instance, variability in APD was observed among 4 regions of the atria (14).

Increased spatial dispersion of the APD in mice facilitated the initiation of AF by anchoring reentrant circuits at border zones of regions with high APD heterogeneity. Although EADs are an important trigger of arrhythmogenesis, EADs failed to initiate AF in the absence of APD heterogeneity. Our findings suggest that persistent $\mathrm{Na}^{+}$current-induced $\mathrm{AF}$ is dependent upon the presence of both EADs and atrial substrate heterogeneity. Computer simulation confirmed that APD heterogeneity is sufficient to sustain rotational reentry upon premature stimulation and to anchor the rotor's center within the boundaries of the APD gradient. It is unlikely that fibrosis, which is only modestly increased in the atria of F1759A-dTG mice, is the major driver of APD heterogeneity or anchoring of the rotors because the distribution of fibrosis is diffuse.

There are several limitations of our study. The best "model" to study human disease is the patient, but in AF, the tissues in which the molecular changes occur that confer and sustain AF are not readily accessible or can be obtained only in the latter stages of the disease process. The difficulties for human research are balanced by the challenges of animal models in which a single or even several animal models of AF can never be representative of all forms of AF. This model of AF relies on the heterogeneous expression of a mutant $\mathrm{Na}_{\mathrm{v}} 1.5$, which confers increased persistent $\mathrm{Na}^{+}$current and heterogeneous prolongation of the APD. Although increased persistent $\mathrm{Na}^{+}$current in atrial cardiomyocytes is associated with AF in humans (2), we cannot conclude that the APD dispersion observed in the LA of humans with $\mathrm{AF}$ is due to heterogeneous, persistent $\mathrm{Na}^{+}$current. Furthermore, although the ex vivo use of ATX-II to increase APD and reduce APD heterogeneity was effective to attenuate the inducibility of AF, its use in vivo is not a viable therapeutic strategy. Ranolazine is FDA approved for chronic angina but is not approved for AF, although recent trials suggest efficacy (28).

In summary, these studies experimentally demonstrate that dispersion of the APD is absolutely required for perpetuation of $\mathrm{AF}$ in a mouse model caused by increased, persistent $\mathrm{Na}^{+}$current. Rotors and wavebreaks, the electrophysiological basis for AF, are anchored to the regions of greatest APD dispersion. Reducing the heterogeneity of the APD, even in the presence of increased EADs, prevented the induction of rotors and wavebreaks and thereby was antifibrillatory. Because spatial APD heterogeneity is present in humans with AF, we propose that identifying regions of APD inhomogeneity may be a novel approach to target key arrhythmic drivers of AF.

\section{Methods}

Animals. All animal experiments were performed according to NIH guidelines. The Institutional Animal Care and Use Committee at Columbia University approved all animal experiments. Both male and female sexes, between 3 and 12 months of age, of the F1759A-Na 1 1.5-TG mouse line (5) and littermate controls were used in this study.

Human studies. Patients undergoing ablation for AF at Columbia University/NewYork-Presbyterian Hospital were enrolled after providing informed consent. The study was approved by the Columbia University IRB. A deflectable 7 Fr MAP catheter (MedFact Engineering) was used. Unipolar data acquisition was performed during atrial pacing at a cycle length of $500 \mathrm{~ms}$. Signal filtering was $0.05-500 \mathrm{~Hz}$ (MAPs), 30-500 Hz (other cardiac signals) and 0.05-100 Hz (ECG). APD was measured at 90\% repolarization during atrial pacing at a cycle length of $500 \mathrm{~ms}$ (120 beats/min) using customized MATLAB software (MathWorks). Three-dimensional, electro-anatomical voltage maps of the LA were obtained using CARTO3 (Biosense Webster) or EnSite Precision (Abbott).

Cellular electrophysiology. Cellular electrophysiology of atrial cardiomyocytes was performed as described (5). Membrane currents from noncontracting, rod-shaped atrial cardiomyocytes with clear striations were measured using whole-cell patch clamp with a MultiClamp 700B amplifier and pCLAMP software (Molecular Devices). The pipette resistance was $0.4-1.0 \mathrm{M} \Omega$ to minimize voltage clamp error. The cell capacitance currents were compensated. Series resistances were compensated at $60 \%$. The leak current was subtracted 
using a $\mathrm{P} / 4$ protocol. The intracellular pipette solution contained (in $\mathrm{mM}$ ): $5 \mathrm{NaCl}, 20 \mathrm{CsCl}, 115 \mathrm{CsF}, 10$ HEPES, and 10 BAPTA ( $\mathrm{pH}$ 7.4) titrated with $\mathrm{CsOH}$. For persistent $\mathrm{Na}^{+}$current determinations, the bath solution contained (in $\mathrm{mM}$ ): $100 \mathrm{NaCl}, 45$ tetraethylammonium chloride (TEA-Cl), $10 \mathrm{HEPES}, 1 \mathrm{MgCl}_{2}$, $0.25 \mathrm{CaCl}_{2}$, and 5 glucose ( $\mathrm{pH} 7.4$ ) titrated with $\mathrm{CsOH}$. In voltage clamp mode, cell membrane potential was held at $-110 \mathrm{mV}$ and stepped to $-30 \mathrm{mV}$ for $190 \mathrm{~ms}$ in the absence and in the presence of ranolazine (200-500 $\mu \mathrm{M})$. The mean values of the currents during the last $10 \mathrm{~ms}$ of the $190 \mathrm{~ms}$ were measured. The difference of these values — "ranolazine-sensitive" current - was used as a measure of persistent $\mathrm{Na}^{+}$current and later normalized to cell capacitance. Peak $\mathrm{Na}^{+}$current density was determined using $5 \mathrm{mM} \mathrm{NaCl}$ in the bath solution. Lidocaine ( $3 \mathrm{mM}$ ) was superfused in the bath solution to determine the lidocaine-resistant current.

Confocal microscopy and immunofluorescence. Isolated atrial cardiomyocytes were fixed for 15 minutes in 4\% paraformaldehyde. Indirect fluorescence was performed using 1:200 rabbit anti-FLAG antibody (F7425, MilliporeSigma) and 1:200 FITC-labeled goat anti-rabbit antibody (F2555, MilliporeSigma). Images were acquired using a confocal microscope (Nikon).

Optical mapping data acquisition and processing. Optical mapping was performed (5) using a complementary metal-oxide semiconductor camera (MICAM Ultima, SciMedia). Movies were acquired at 1000 frames/s for $4-5$ seconds, with $100 \times 100$ pixel resolution $(0.095 \mathrm{~mm} / \mathrm{pixel})$. Images were processed using custom software Precision Visuals Workstation Analysis and Visualization Environment (Visual Numerics, Inc.) (30). Dominant frequency and phase maps were obtained in AF, and APD and conduction velocity maps were obtained with atrial pacing at $10 \mathrm{~Hz}$. Average APD, maximum APD, and APD dispersion $\left(A P D_{\max }-\mathrm{APD}_{\min }\right)$ were calculated for the whole atrium and compared between the regions of highest SPD to a neighboring $10 \times 10$ pixel area (30). High APD gradients were defined as regions where the difference between a long APD and a neighboring short APD within a $10 \times 10$ pixel area was greatest. Rotational activity of at least 1 cycle was classified as a rotor (30). SPD maps were created by overlaying the distribution of singularity points from the phase movie for approximately 4.1 seconds. The high SPD regions were defined as SPD greater than 30\% compared with surrounding pixels (30). Pacing-induced AF was assessed by 3 attempts of burst pacing at twice the excitation threshold of the left atrium $(20 \mathrm{~Hz}$, amplitude 0.5-2.0 mA, $5 \mathrm{~ms}$ ). SEA-0400 (Chemscene Chemicals), ranolazine (Alomone), ATX-II (MilliporeSigma), and digoxin (West-Ward Pharmaceuticals) were retrograde perfused via the aortic cannula.

Computational modeling. A cellular automaton was used to model atrial fibrillatory activity (16). A grid consisting of $576 \times 576$ nodes was used for analysis. The distance between nodes was set at $1 \mathrm{~mm}$, and the time step was $2 \mathrm{~ms}$. Each node was programmed to 1 of 3 states: (a) activating; (b) recovering from activation, such as being in the refractory state; or (c) recovered from activation and excitable. State 1 was set to $2 \mathrm{~ms}$, while state $23 / 4$, the refractory period or APD, was varied throughout the grid. The conduction velocity for wavefront propagation was set to $0.5 \mathrm{~mm} / \mathrm{ms}$ along the vertical axis and $0.25 \mathrm{~mm} / \mathrm{ms}$ along the horizontal axis (anisotropic ratio of 2:1).

Statistics. Group data are presented as mean \pm SEM. Statistical comparisons between the groups were tested using an unpaired, 2-tailed Student's $t$ test and 1-way ANOVA tests for multiple comparisons. A $P$ value less than 0.05 was considered statistically significant. All statistical analyses were performed with GraphPad Prism 6.0.

Study approval. Human subject research was approved by the Columbia University IRB, Columbia University Human Research Protection Office, New York. All subjects provided informed consent before their participation in the study.

\section{Author contributions}

UMRA, SOM, ARM, and EYW designed the study. EYW, UMRA, JA, AK, SZ, SM, JB, DR, AG, MW, VI, DS, EJC, and LY performed experiments and collected the data. UMRA, HG, ARM, SOM, and EYW analyzed the data. UMRA, SOM, and EYW wrote the manuscript.

\section{Acknowledgments}

The authors thank Igor Efimov (George Washington University) for generous technical assistance with the optical imaging experiments and analysis. The authors thank José Jalife (University of Michigan) and James Coromilas (Robert Wood Johnson Medical School and Rutgers Biomedical and Health Sciences) for helpful discussions and suggestions. We thank Sadiq Khan, Kristin Pallister, and Pierre Nauleau for technical assistance. We thank Angelo Biviano for help with MAP recordings. The research was supported by National Heart, Lung, and Blood Institute R01HL122967 and R01HL123483 to SOM and a gift from 
the Izard family to EYW. EYW was supported by NIH K08HL122526, the Louis V. Gerstner, Jr., Scholars Program; the Esther Aboodi Endowed Professorship at Columbia University; and the M. Iréne Ferrer Scholar Award from the Foundation of Gender-Specific Medicine.

Address correspondence to: Elaine Y. Wan or Steven O. Marx, 622 W168 St., PH 3-Center, New York, New York 10032, USA. Phone: 212.342.0400; Email: eyw2003@cumc.columbia.edu (EYW). Phone: 212.305.0271; Email: sm460@cumc.columbia.edu (SOM).

1. Moss AJ, Kass RS. Long QT syndrome: from channels to cardiac arrhythmias. J Clin Invest. 2005;115(8):2018-2024

2. Sossalla $\mathrm{S}$, et al. Altered $\mathrm{Na}(+)$ currents in atrial fibrillation effects of ranolazine on arrhythmias and contractility in human atrial myocardium. J Am Coll Cardiol. 2010;55(21):2330-2342.

3. Toischer K, et al. Role of late sodium current as a potential arrhythmogenic mechanism in the progression of pressure-induced heart disease. J Mol Cell Cardiol. 2013;61:111-122.

4. Signore $\mathrm{S}$, et al. Late $\mathrm{Na}(+)$ current and protracted electrical recovery are critical determinants of the aging myopathy. Nat Commun. 2015;6:8803

5. Wan E, et al. Aberrant sodium influx causes cardiomyopathy and atrial fibrillation in mice. J Clin Invest. 2016;126(1):112-122.

6. Wang YL, et al. Optimal endpoint for catheter ablation of longstanding persistent atrial fibrillation: A randomized clinical trial. Pacing Clin Electrophysiol. 2018;41(2):172-178.

7. Narayan SM, Krummen DE, Shivkumar K, Clopton P, Rappel WJ, Miller JM. Treatment of atrial fibrillation by the ablation of localized sources: CONFIRM (Conventional Ablation for Atrial Fibrillation With or Without Focal Impulse and Rotor Modulation) trial. J Am Coll Cardiol. 2012;60(7):628-636.

8. Cochet $\mathrm{H}$, et al. Relationship between fibrosis detected on late gadolinium-enhanced cardiac magnetic resonance and re-entrant activity assessed with electrocardiographic imaging in human persistent atrial fibrillation. JACC Clin Electrophysiol. 2018;4(1):17-29.

9. Chrispin J, et al. Lack of regional association between atrial late gadolinium enhancement on cardiac magnetic resonance and atrial fibrillation rotors. Heart Rhythm. 2016;13(3):654-660.

10. Sohns $\mathrm{C}$, et al. First-in-man analysis of the relationship between electrical rotors from noninvasive panoramic mapping and atrial fibrosis from magnetic resonance imaging in patients with persistent atrial fibrillation. Circ Arrhythm Electrophysiol. 2017;10(8):e004419.

11. Campbell K, Calvo CJ, Mironov S, Herron T, Berenfeld O, Jalife J. Spatial gradients in action potential duration created by regional magnetofection of $\mathrm{hERG}$ are a substrate for wavebreak and turbulent propagation in cardiomyocyte monolayers. $J$ Physiol (Lond). 2012;590(24):6363-6379.

12. Seitz J, et al. AF ablation guided by spatiotemporal electrogram dispersion without pulmonary vein isolation: a wholly patient-tailored approach. J Am Coll Cardiol. 2017;69(3):303-321.

13. Qu Z, Garfinkel A, Chen PS, Weiss JN. Mechanisms of discordant alternans and induction of reentry in simulated cardiac tissue. Circulation. 2000;102(14):1664-1670.

14. Al Abed A, Lovell NH, Dokos S. Local heterogeneous electrical restitution properties of rabbit atria. J Cardiovasc Electrophysiol. 2016;27(6):743-753.

15. Franz MR. Current status of monophasic action potential recording: theories, measurements and interpretations. Cardiovasc Res. 1999;41(1):25-40

16. Ciaccio EJ, Peters NS, Garan H. Effects of refractory gradients and ablation on fibrillatory activity. Comput Biol Med. 2018;95:175-187

17. Sanbe A, Gulick J, Hanks MC, Liang Q, Osinska H, Robbins J. Reengineering inducible cardiac-specific transgenesis with an attenuated myosin heavy chain promoter. Circ Res. 2003;92(6):609-616.

18. Valencik ML, McDonald JA. Codon optimization markedly improves doxycycline regulated gene expression in the mouse heart. Transgenic Res. 2001;10(3):269-275.

19. McNulty MM, Edgerton GB, Shah RD, Hanck DA, Fozzard HA, Lipkind GM. Charge at the lidocaine binding site residue Phe-1759 affects permeation in human cardiac voltage-gated sodium channels. $J$ Physiol (Lond). 2007;581(Pt 2):741-755.

20. Narayan SM, Franz MR, Clopton P, Pruvot EJ, Krummen DE. Repolarization alternans reveals vulnerability to human atrial fibrillation. Circulation. 2011;123(25):2922-2930.

21. Du C, Zhang Y, El Harchi A, Dempsey CE, Hancox JC. Ranolazine inhibition of hERG potassium channels: drug-pore interac tions and reduced potency against inactivation mutants. J Mol Cell Cardiol. 2014;74:220-230.

22. Catterall WA, Cestèle S, Yarov-Yarovoy V, Yu FH, Konoki K, Scheuer T. Voltage-gated ion channels and gating modifier toxins. Toxicon. 2007;49(2):124-141.

23. Saha M, et al. Wavelength and fibrosis affect phase singularity locations during atrial fibrillation. Front Physiol. 2018;9:1207.

24. Varela M, Colman MA, Hancox JC, Aslanidi OV. Atrial heterogeneity generates re-entrant substrate during atrial fibrillation and anti-arrhythmic drug action: mechanistic insights from canine atrial models. PLoS Comput Biol. 2016;12(12):e1005245.

25. Remme CA. Cardiac sodium channelopathy associated with SCN5A mutations: electrophysiological, molecular and genetic aspects. J Physiol (Lond). 2013;591(17):4099-4116.

26. Benito B, et al. A mutation in the sodium channel is responsible for the association of long QT syndrome and familial atrial fibrillation. Heart Rhythm. 2008;5(10):1434-1440.

27. Scirica BM, et al. Effect of ranolazine, an antianginal agent with novel electrophysiological properties, on the incidence of arrhythmias in patients with non ST-segment elevation acute coronary syndrome: results from the Metabolic Efficiency With Ranolazine for Less Ischemia in Non ST-Elevation Acute Coronary Syndrome Thrombolysis in Myocardial Infarction 36 (MERLIN-TIMI 36) randomized controlled trial. Circulation. 2007;116(15):1647-1652.

28. Reiffel JA, et al. The HARMONY Trial: combined ranolazine and dronedarone in the management of paroxysmal atrial fibril- 
lation: mechanistic and therapeutic synergism. Circ Arrhythm Electrophysiol. 2015;8(5):1048-1056.

29. Kondo M, Nesterenko V, Antzelevitch C. Cellular basis for the monophasic action potential. Which electrode is the recording electrode? Cardiovasc Res. 2004;63(4):635-644.

30. Yamazaki M, et al. Acute regional left atrial ischemia causes acceleration of atrial drivers during atrial fibrillation. Heart Rhythm. 2013;10(6):901-909. 